\title{
All-Loop Singularities of Scattering Amplitudes in Massless Planar Theories
}

\author{
Igor Prlina, ${ }^{1}$ Marcus Spradlin, ${ }^{1,2}$ and Stefan Stanojevic ${ }^{1}$ \\ ${ }^{1}$ Department of Physics, Brown University, Providence, Rhode Island 02912, USA \\ ${ }^{2}$ School of Natural Sciences, Institute for Advanced Study, Princeton, New Jersey 08540, USA
}

(Received 29 May 2018; published 21 August 2018)

\begin{abstract}
In massless quantum field theories the Landau equations are invariant under graph operations familiar from the theory of electrical circuits. Using a theorem on the $Y-\Delta$ reducibility of planar circuits we prove that the set of first-type Landau singularities of an $n$-particle scattering amplitude in any massless planar theory, at any finite loop order, is a subset of those of a certain $n$-particle $\left\lfloor(n-2)^{2} / 4\right\rfloor$-loop "ziggurat" graph. We determine this singularity locus explicitly for $n=6$ and find that it corresponds precisely to the vanishing of the symbol letters familiar from the hexagon bootstrap in supersymmetric Yang-Mills (SYM) theory. Further implications for SYM theory are discussed.
\end{abstract}

DOI: 10.1103/PhysRevLett.121.081601

Introduction.-For over half a century, much has been learned from the study of singularities of scattering amplitudes in quantum field theory, an important class of which are encoded in the Landau equations [1]. This Letter combines two simple statements to arrive at a general result about such singularities. The first is based on the long appreciated and exploited analogy between Feynman diagrams and electrical circuits [2-5]. In massless field theories, the sets of solutions to the Landau equations are invariant under the elementary graph operations familiar from circuit theory, including the $Y-\Delta$ transformation that replaces a triangle subgraph with a trivalent vertex or vice versa. The second is a theorem of Gitler [6], who proved that all relevant (specified below) planar graphs can be $Y-\Delta$ reduced to a class we call ziggurats (Fig. 2).

We conclude that the $n$-particle $\left\lfloor(n-2)^{2} / 4\right\rfloor$-loop ziggurat graph encodes all possible first-type Landau singularities of any $n$-particle amplitude at any finite loop order in any massless planar theory. In Sec. VI, we discuss several interesting implications of our result for planar $\mathcal{N}=4$ supersymmetric Yang-Mills (SYM) theory, which provided the motivation for this work [7-10].

Landau graphs and singularities.-The Landau equations encapsulate the singularity structure of scattering amplitudes via Landau graphs. In planar quantum field theories, the exclusive focus of this Letter, we need only consider plane graphs. An $L$-loop $m$-point plane Landau graph is a plane graph with $L+1$ faces and $m$ distinguished vertices, called terminals, that lie on a common

Published by the American Physical Society under the terms of the Creative Commons Attribution 4.0 International license. Further distribution of this work must maintain attribution to the author(s) and the published article's title, journal citation, and DOI. Funded by SCOAP . face called the unbounded face. Henceforth, we use "vertex" only for those that are not terminals, and "face" only for the $L$ faces that are not the unbounded face.

Each edge $j$ is assigned a four-momentum vector $q_{j}$, the analog of electric current. At each vertex, the vector sum of incoming momenta equals that of the outgoing momenta (current conservation). This constraint is not applied at terminals, which are the locations where a circuit can be probed by connecting external sources or sinks of current. In field theory, these correspond to the momenta carried by incoming or outgoing particles. If we label the terminals $a=1, \ldots, m$ (in cyclic order around the unbounded face) and let $P_{a}$ denote the four-momentum flowing into the graph at terminal $a$, then energy-momentum conservation requires that $\sum_{a} P_{a}=0$, and it implies that precisely $L$ of the $q_{j}$ 's are linearly independent.

Our interest lies in understanding the loci in $P_{a}$-space on which amplitudes may have singularities. A Landau graph is said to have Landau singularities of the first type (LS) at values of $P_{a}$ for which the Landau equations [1]

$$
\begin{gathered}
\alpha_{j} q_{j}^{2}=0 \quad \text { for each edge } j, \quad \text { and } \\
\sum_{\text {edges } j \in \mathcal{F}} \alpha_{j} q_{j}=0 \text { for each face } \mathcal{F}
\end{gathered}
$$

admit solutions for the Feynman parameters $\alpha_{j}$ (omitting the trivial solution where all $\alpha_{j}=0$ ). In Eq. (1), we omitted a term proportional to $m_{j}^{2}$ that would be present in massive theories.

The Landau equations generally admit several branches of solutions. The leading LS of a graph $\mathcal{G}$ are those associated to branches having $q_{j}^{2}=0$ for all $j$ (regardless of whether any of the $\alpha_{j}$ 's are zero). LS associated to branches on which one or more of the $q_{j}^{2}$ are not zero (in which case the 
(a)
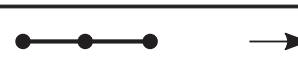

(b)

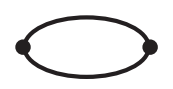

(c)
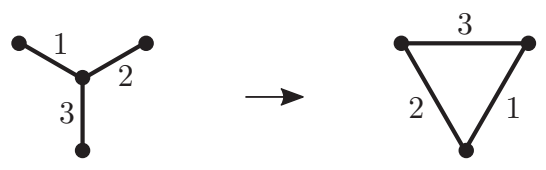

FIG. 1. Elementary circuit moves that preserve solution sets of the massless Landau equations: (a) series reduction, (b) parallel reduction, and (c) $Y-\Delta$ reduction.

corresponding $\alpha_{j}$ 's must necessarily vanish) can be interpreted as leading singularities of a relaxed graph, obtained from $\mathcal{G}$ by contracting the edges associated to the vanishing $\alpha_{j}$ 's.

A graph is called $c$ connected if it remains connected after the removal of any $c-1$ vertices. The set of LS for a 1-connected graph is the union of those associated to each 2-connected component since the Landau equations completely decouple. Therefore, we can confine our attention to 2-connected graphs.

Elementary circuit operations.-We call Eq. (2) the Kirchhoff conditions in recognition of their circuit analog where the $\alpha_{j}$ 's play the role of resistances. The analog of the on shell conditions (1) is mysterious, but a remarkable feature of massless theories is that: The graph moves that are familiar from elementary electrical circuit theory preserve the solution sets of Eqs. (1) and (2), and hence, the sets of LS in any massless field theory.

Series reduction [Fig. 1(a)] removes any vertex of degree two. Since $q_{2}=q_{1}$ by momentum conservation, the Landau equations are trivially preserved if the two edges with Feynman parameters $\alpha_{1}, \alpha_{2}$ are replaced by a single edge carrying momentum $q^{\prime}=q_{1}=q_{2}$ and Feynman parameter $\alpha^{\prime}=\alpha_{1}+\alpha_{2}$.

Parallel reduction [Fig. 1(b)] collapses any bubble subgraph. It is easy to verify (see e.g., Appendix A.1 of [8]) the Landau equations are preserved if the two edges of the bubble are replaced by a single edge carrying momentum $q^{\prime}=$ $q_{1}+q_{2}$ and Feynman parameter $\alpha^{\prime}=\alpha_{1} \alpha_{2} /\left(\alpha_{1}+\alpha_{2}\right)$.

The $Y-\Delta$ reduction [Fig. 1(c)] replaces a vertex of degree three (a " $Y$ ") with a triangle subgraph (a " $\Delta$ ") or vice versa. Generically, the Feynman parameters $\alpha_{i}$ of the $\Delta$ are related to those of the $Y$, which we call $\beta_{i}$, by

$$
\beta_{1}=\frac{\alpha_{2} \alpha_{3}}{\alpha_{1}+\alpha_{2}+\alpha_{3}}, \quad \text { and cyclic. }
$$

On branches where one or more of the parameters vanish, this relation must be suitably modified. For example, if a branch of solutions for a graph containing a $Y$ has $\beta_{1}=$ $\beta_{2}=0$ but $\beta_{3}$ nonzero, then the corresponding branch for the reduced graph has $\alpha_{3}=0$ but $\alpha_{1}, \alpha_{2}$ nonzero.

The invariance of the Kirchhoff conditions (2) under $Y-\Delta$ reduction follows straightforwardly from these Feynman
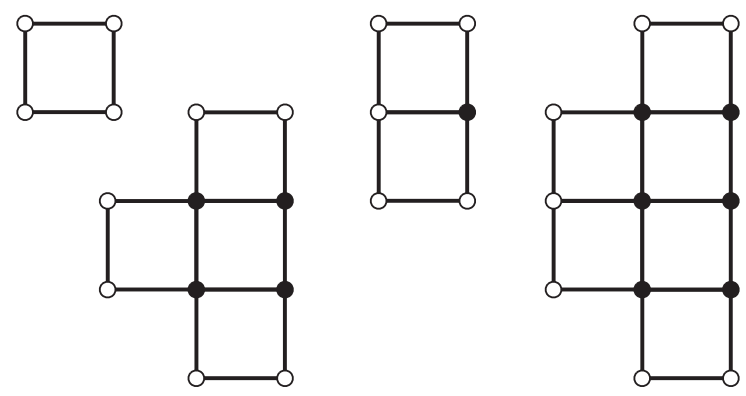

FIG. 2. The four-, six-, five-, and seven-terminal ziggurat graphs. The open circles are terminals, and the filled circles are vertices. The pattern continues in the obvious way, but note, there is an essential difference between ziggurat graphs with an even or odd number of terminals in that only the latter have a terminal of degree three.

parameter assignments. The invariance of the on shell conditions (1) is nontrivial, and it follows from the analysis in Appendix A.2 of [8].

The proof of the crucial theorem of [6] that we employ in the next section relies on three additional, relatively simple moves, that trivially preserve the essential content of the Landau equations. These are (d) the deletion of a "tadpole" (edges that connect a vertex or terminal to itself), (e) the deletion of a "hanging propagator" (a vertex of degree one and the edge connected to it), and (f) the contraction of an edge connected to a terminal of degree one (called "FP assignment" [11]). The last of these is, strictly speaking, not completely trivial at the level of the Landau equations; it just removes an otherwise uninteresting bubble singularity.

Reduction of planar graphs.-The reduction of graphs under circuit operations is a well-studied problem in the mathematical literature. When it is declared that a certain subset of vertices are to be considered terminals (which may not be removed by series or $Y$ - $\Delta$ reduction) the corresponding problem is called terminal $Y-\Delta$ reducibility. Aspects of this problem have been studied in [11-16], including an application to Feynman diagrams in [17]. For our purpose, the key result comes from the Ph.D. thesis of I. Gitler [6], who proved that any planar 2-connected graph with $m$ terminals lying on the same face can be reduced to a graph of the kind shown in Fig. 2, which we call ziggurat graphs, or to a minor thereof. We denote the $m$-terminal ziggurat graph by $\mathcal{T}_{m}$, and note that, a minor of a graph $\mathcal{G}$ is any graph that can be obtained from $\mathcal{G}$ by a sequence of edge contractions and/or edge deletions.

An edge contraction corresponds to a relaxation, while an edge deletion corresponds to setting the associated $q_{j}$ to zero. Therefore, the LS associated to any minor of a graph $\mathcal{G}$ are a subset of those associated to $\mathcal{G}$. Consequently, we do not need to explicitly enumerate all minors of $\mathcal{T}_{m}$; their LS are already contained in the set of singularities of $\mathcal{T}_{m}$ itself.

It is conventional to discuss scattering amplitudes for a fixed number $n$ of external particles, each of which carries some momentum $p_{i}$ that in massless theories satisfies 


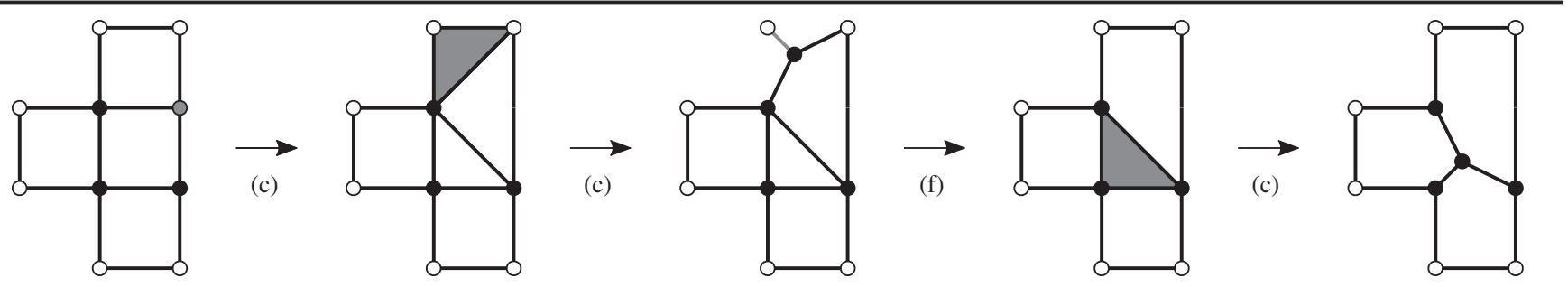

FIG. 3. The six-terminal ziggurat graph can be reduced to a three-loop graph by a sequence of three $Y$ - $\Delta$ reductions and one FP assignment. In each case the vertex, edge, or face to be transformed is highlighted in gray.

$p_{i}^{2}=0$. These individual particles are denoted graphically by attaching a total of $n$ external edges to the terminals, with at least one per terminal. Any graph with $m \leq n$ terminals is potentially relevant to finding the Landau singularities of an $n$-particle amplitude. However, if $m<n$, then $\mathcal{T}_{m}$ is a minor of $\mathcal{T}_{n}$, so the LS of the former are a subset of those of the latter. Therefore, to find the LS of an $n$-particle amplitude, it suffices to find those of the $n$-terminal ziggurat graph $\mathcal{T}_{n}$ with precisely one external edge attached to each terminal. We call this the $n$-particle ziggurat graph and finally summarize: The first-type Landau singularities of an $n$-particle scattering amplitude in any massless planar field theory are a subset of those of the $n$-particle ziggurat graph.

While the LS of the ziggurat graph exhaust the set of singularities that may appear in any massless planar theory, we cannot rule out the possibility that in certain special theories, the actual set of singularities may be smaller because of a cancellation between the contributions of different graphs to a given amplitude. We discuss this further in Sec. VI.

The $Y-\Delta$ reduction changes (and generally reduces) the number of faces of a graph, so the above statement does not hold at a fixed loop order $L$; rather it is an all-order relation about the full set of LS of $n$-particle amplitudes. Since the $n$-particle ziggurat graph has $L=\left\lfloor(n-2)^{2} / 4\right\rfloor$ faces, a single computation at $\left\lfloor(n-2)^{2} / 4\right\rfloor$-loop order suffices to expose all possible Landau singularities of any $n$-particle amplitude.

This bound is unnecessarily high. Gitler's theorem does not imply that ziggurat graphs cannot be reduced to graphs of a lower loop order, and in general, this is possible: Figure 3 shows that $\mathcal{T}_{6}$ can be reduced to a three-loop wheel graph whose 6-particle avatar we show in Fig. 4. Ziggurat graphs with more than six terminals can also be further reduced, but we have not found a lower bound on the loop order that can be obtained for a general $n$.

Landau analysis of the wheel.-Here, we analyze the Landau equations for the graph shown in Fig. 4. The six external edges carry momenta $p_{1}, \ldots, p_{6}$ subject to $\sum_{i} p_{i}=0$ and $p_{i}^{2}=0$ for each $i$. Using momentum conservation at each vertex, the momentum $q_{j}$ carried by each internal edge can be expressed in terms of the $p_{i}$ and three other linearly independent momenta, which we take to be $l_{r}$, for $r=1,2,3$, as shown in the figure. Initially, we consider the leading LS, for which we impose the on shell conditions

$$
\begin{aligned}
\left(l_{1}-p_{1}\right)^{2}=l_{1}^{2}=\left(l_{1}+p_{2}\right)^{2} & =0, \\
\left(l_{2}-p_{3}\right)^{2}=l_{2}^{2}=\left(l_{2}+p_{4}\right)^{2} & =0, \\
\left(l_{3}-p_{5}\right)^{2}=l_{3}^{2}=\left(l_{3}+p_{6}\right)^{2} & =0, \\
\left(l_{1}+p_{2}-l_{2}+p_{3}\right)^{2} & =0, \\
\left(l_{2}+p_{4}-l_{3}+p_{5}\right)^{2} & =0, \\
\left(l_{3}+p_{6}-l_{1}+p_{1}\right)^{2} & =0 .
\end{aligned}
$$

For generic $p_{i}$, there are 16 discrete solutions for the $l_{r}$ 's, which we denote by $l_{r}^{*}\left(p_{i}\right)$. To enumerate these solutions, it is technically helpful to use momentum twistor variables [18], in which case the solutions can be associated with on shell diagrams [19]. Although the analysis is still applicable to general massless planar theories, in the special context of SYM theory, two cut solutions have MHV support, twelve NMHV, and two NNMHV.

The Kirchhoff conditions are

$$
\begin{aligned}
0= & \alpha_{1}\left(l_{1}-p_{1}\right)+\alpha_{2} l_{1}+\alpha_{3}\left(l_{1}+p_{2}\right) \\
& +\alpha_{10}\left(l_{3}+p_{6}-l_{1}+p_{1}\right)+\alpha_{11}\left(l_{1}+p_{2}-l_{2}+p_{3}\right), \\
0= & \alpha_{4}\left(l_{2}-p_{3}\right)+\alpha_{5} l_{2}+\alpha_{6}\left(l_{2}+p_{4}\right) \\
& +\alpha_{11}\left(l_{1}+p_{2}-l_{2}+p_{3}\right)+\alpha_{12}\left(l_{2}+p_{4}-l_{3}+p_{5}\right), \\
0= & \alpha_{7}\left(l_{3}-p_{5}\right)+\alpha_{8} l_{3}+\alpha_{9}\left(l_{3}+p_{6}\right) \\
& +\alpha_{12}\left(l_{2}+p_{4}-l_{3}+p_{5}\right)+\alpha_{10}\left(l_{3}+p_{6}-l_{1}+p_{1}\right) .
\end{aligned}
$$

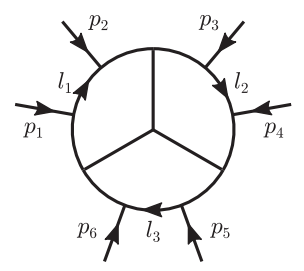

FIG. 4. The three-loop six-particle wheel graph. The leading LS of this graph exhaust all possible LS of six-particle amplitudes in any massless planar field theory, to any finite loop order. 
Nontrivial solutions to this $12 \times 12$ linear system exist only if the associated Kirchhoff determinant $K\left(p_{i}, l_{r}\right)$ vanishes. By evaluating $K$ on each solution $l_{r}=l_{r}^{*}\left(p_{i}\right)$, the condition for the existence of a nontrivial solution to the Landau equations can be expressed entirely in terms of the external momenta. Using

$u=\frac{s_{12} s_{45}}{s_{123} s_{345}}, \quad v=\frac{s_{23} s_{56}}{s_{234} s_{123}}, \quad w=\frac{s_{34} s_{61}}{s_{345} s_{234}}$,

where $s_{i \ldots j}=\left(p_{i}+\cdots+p_{j}\right)^{2}$, we find that $K\left(p_{i}, l_{r}^{*}\left(p_{i}\right)\right)=0$ can only be satisfied if an element of

$$
S=\left\{u, v, w, 1-u, 1-v, 1-w, \frac{1}{u}, \frac{1}{v}, \frac{1}{w}\right\}
$$

vanishes. Therefore, the three-loop six-particle wheel graph has LS on the locus

$$
\mathcal{S}=\bigcup_{s \in S}\{s=0\}
$$

It is straightforward to analyze all subleading LS corresponding to relaxations. We refer the reader to $[7,8,10]$ where this type of analysis has been carried out in detail in several examples. We find no additional LS beyond those that appear at leading order. We conclude that the LS of any six-particle amplitude in any massless planar field theory, at any finite loop order, are given by Eqs. (7) and (8), or a proper subset thereof.

Second-type singularities.-The LS studied here do not exhaust all possible singularities of amplitudes in quantum field theories. There also exist "second-type" singularities [20,21], sometimes called "non-Landauian" [22]. These arise in loop integrals as pinch singularities at infinite loop momentum, and they are encoded in a modified version of Eqs. (1) and (2).

In the next section, we consider the special case of the SYM theory, which possesses a remarkable dual conformal symmetry [23-25], implying that there is no invariant notion of "infinity" in momentum space. We therefore expect that second-type singularities should be absent in any dual conformal invariant theory [7]. Because ziggurat graphs are manifestly dual conformal invariant, the LS of the ziggurat graphs should capture the entire "dual conformally invariant part" of the singularity structure of all massless planar theories; this means the singularity loci that do not involve the infinity twistor.

Planar SYM theory.--In Sec. III, we acknowledged that in certain theories, the actual set of singularities of amplitudes may be strictly smaller than that of the ziggurat graphs due to cancellations. Contrary to the expectation that SYM theory might seem the most promising candidate to exhibit such cancellations, we now argue that: Perturbative amplitudes in SYM theory exhibit first-type Landau singularities on all such loci that are possible in any massless planar field theory.
Our results suggest that this statement is true separately in each helicity sector. Specifically, for any fixed $n$ and any $0 \leq k \leq n-4$, there is a finite value of $L_{n, k}$, such that the singularity locus of the $L$-loop $n$-particle $\mathrm{N}^{k} \mathrm{MHV}$ amplitude is identical to that of the $n$-particle ziggurat graph for all $L \geq L_{n, k}$. In order to verify this claim, it suffices to construct an $n$-particle on shell diagram with $\mathrm{N}^{k} \mathrm{MHV}$ support that has the same LS as the n-particle ziggurat graph; or equivalently, to write down a corresponding configuration of lines inside the amplituhedron [26] $\mathcal{A}_{n, k, L}$ for some sufficiently high $L$.

To see that this is plausible, note that, the appearance of a given singularity at some fixed $k$ and $L$ implies the existence of the same singularity at lower $k$ but higher $L$ by performing the opposite of parallel reductions_-doubling one or more edges of the relevant graph to make bubbles. For example, while one-loop MHV amplitudes do not have singularities of three-mass box type, two-loop MHV amplitudes do [27]. Similarly, while two-loop MHV amplitudes do not have singularities of the four-mass box type, we expect that three-loop MHV and two-loop NMHV amplitudes do. (Our analysis is silent on the question of whether the symbol alphabets of these amplitudes contain square roots; see Sec. VII of [9].)

It is simple to convert the $n$-particle ziggurat graph into a valid on shell diagram with MHV support by doubling each internal edge into a bubble. In this way, it is easy to write an explicit mutually positive configuration of lines inside the MHV amplituhedron. While this construction suffices to demonstrate the claim, it is overkill; we expect MHV support to be reached at a much lower loop level than this argument would require, as can be checked on a case by case basis for a small $n$.

Symbol alphabets. - Let us comment on the connection of our work to symbol alphabets. All of the singularities tabulated in Eq. (8) are known to appear in both MHV and NMHV six-particle amplitudes, starting at the two-loop order $[28,29]$. Indeed, the hypothesis that there are no new singularity loci at any higher loop order (which we now consider to be proven) underlies a bootstrap program that has made it possible for impressive explicit computations of six-particle amplitudes in SYM theory [29-35]. An analogous program for $n=7$ has allowed for the computation of symbols of seven-particle amplitudes [36,37].

The hexagon bootstrap involves, in addition to the quantities appearing in Eq. (7), three particular algebraic functions $y_{u}, y_{v}, y_{w}$ that also vanish only on the same locus $\mathcal{S}$. This highlights the fact that the connection between LS and symbol alphabets is somewhat indirect. Knowledge of the former tells us about the locus where symbol letters vanish [38] or have branch points (see Sec. VII of [9]). In order to determine what the symbol letters of an amplitude actually are away from these loci, it seems necessary to invoke some other kind of structure; cluster algebras may have a role to play here $[39,40]$. 
Conclusion.-We leave several questions for future work. What is the minimum loop order to which the $n$-particle ziggurat graph can be reduced? Can one characterize LS for an arbitrary $n$, generalizing the result for $n=6$ in Sec. IV? It is possible to classify second-type singularities, even if only in certain theories? The graph moves reviewed in Sec. II preserve the LS even for nonplanar graphs; are there results on nonplanar $Y-\Delta$ reducibility (see e.g., $[41,42]$ ) that may be useful for nonplanar (but still massless) theories?

The ziggurat graphs, and those to which they can be reduced, might warrant further study for their own sake. They generalize those studied in $[43,44]$, and they are particular cases of the graphs that have attracted recent interest, e.g., in $[45,46]$, in the context of "fishnet" theories. We have only looked at their singularity loci; it would be interesting to explore the structure of their cuts, perhaps in connection with the coaction studied in [47-51].

In SYM theory, the technology might exist to address more detailed questions. For a general $n$ and $k$, what is the minimum loop order at which the Landau singularities of the $n$-particle $\mathrm{N}^{k} \mathrm{MHV}$ amplitude saturate? Is there a direct connection between Landau singularities, ziggurat graphs, and cluster algebras? For amplitudes of a generalized polylogarithm type, what are the actual symbol letters for a general $n, k$, and loop order? How do LS manifest themselves in general amplitudes that are of more complicated functional type?.

We are grateful to C. Colbourn for correspondence, to N. Arkani-Hamed for stimulating discussions, to J. Bourjaily for helpful comments on the draft, and to T. Dennen, J. Stankowicz, and A. Volovich for collaboration on closely related work. We are especially indebted to I. Gitler for sending us the relevant portion of his Ph.D. thesis. This work was supported in part by the US Department of Energy under contract No. DE-SC0010010 Task A and the Simons Fellowship Program in Theoretical Physics (MS).

[1] L. D. Landau, Nucl. Phys. 13, 181 (1959).

[2] J. Mathews, Phys. Rev. 113, 381 (1959).

[3] T. T. Wu, Phys. Rev. 123, 678 (1961).

[4] T. T. Wu, Phys. Rev. 123, 689 (1961).

[5] J. Bjorken and S. Drell, Relativistic Quantum Fields (McGraw-Hill, New York, 1965).

[6] I. Gitler, Delta-Wye-Delta Transformations: Algorithms and Applications," Ph.D. Thesis, University of Waterloo, 1991.

[7] T. Dennen, M. Spradlin, and A. Volovich, J. High Energy Phys. 03 (2016) 069.

[8] T. Dennen, I. Prlina, M. Spradlin, S. Stanojevic, and A. Volovich, J. High Energy Phys. 06 (2017) 152.

[9] I. Prlina, M. Spradlin, J. Stankowicz, S. Stanojevic, and A. Volovich, J. High Energy Phys. 05 (2018) 159.

[10] I. Prlina, M. Spradlin, J. Stankowicz, and S. Stanojevic, J. High Energy Phys. 04 (2018) 049.

[11] I. Gitler and F. Sagols, Network 57, 174 (2011).
[12] S. B. Akers, Oper. Res. 8, 311 (1960).

[13] T. A. Feo and J. S. Provan, Oper. Res. 41, 572 (1993).

[14] Y. C. Verdière, I. Gitler, and D. Vertigan, Commentarii mathematici Helvetici 71, 144 (1996).

[15] D. Archdeacon, C. J. Colbourn, I. Gitler, and J. S. Provan, J. Graph Theory 33, 83 (2000).

[16] L. Demasi and B. Mohar, Proceedings of the Twenty-Sixth Annual ACM-SIAM Symposium on Discrete Algorithms, San Diego, California, 2015 (SIAM, Philadelphia, 2015) pp. $1728-1742$.

[17] A. T. Suzuki, Can. J. Phys. 92, 131 (2014).

[18] A. Hodges, J. High Energy Phys. 05 (2013) 135.

[19] N. Arkani-Hamed, J. L. Bourjaily, F. Cachazo, A. B. Goncharov, A. Postnikov, and J. Trnka, arXiv:1212.5605.

[20] D. B. Fairlie, P. V. Landshoff, J. Nuttall, and J. C. Polkinghorne, J. Math. Phys. 3, 594 (1962).

[21] R. J. Eden, P. V. Landshoff, D. I. Olive, and J. C. Polkinghorne, The Analytic S-Matrix (Cambridge University Press, Cambridge, England, 1966).

[22] R. E. Cutkosky, J. Math. Phys. 1, 429 (1960).

[23] J. M. Drummond, J. Henn, V. A. Smirnov, and E. Sokatchev, J. High Energy Phys. 01 (2007) 064.

[24] L. F. Alday and J. M. Maldacena, J. High Energy Phys. 06 (2007) 064.

[25] J. M. Drummond, J. Henn, G. P. Korchemsky, and E. Sokatchev, Nucl. Phys. B828, 317 (2010).

[26] N. Arkani-Hamed and J. Trnka, J. High Energy Phys. 10 (2014) 030.

[27] S. Caron-Huot, J. High Energy Phys. 12 (2011) 066.

[28] A. B. Goncharov, M. Spradlin, C. Vergu, and A. Volovich, Phys. Rev. Lett. 105, 151605 (2010).

[29] L. J. Dixon, J. M. Drummond, and J. M. Henn, J. High Energy Phys. 01 (2012) 024.

[30] L. J. Dixon, J. M. Drummond, and J. M. Henn, J. High Energy Phys. 11 (2011) 023.

[31] L. J. Dixon, J. M. Drummond, M. von Hippel, and J. Pennington, J. High Energy Phys. 12 (2013) 049.

[32] L. J. Dixon, J. M. Drummond, C. Duhr, M. von Hippel, and J. Pennington, Proc. Sci., LL2014 (2014) 077.

[33] L. J. Dixon and M. von Hippel, J. High Energy Phys. 10 (2014) 065.

[34] L. J. Dixon, M. von Hippel, and A. J. McLeod, J. High Energy Phys. 01 (2016) 053.

[35] S. Caron-Huot, L. J. Dixon, A. McLeod, and M. von Hippel, Phys. Rev. Lett. 117, 241601 (2016).

[36] J. M. Drummond, G. Papathanasiou, and M. Spradlin, J. High Energy Phys. 03 (2015) 072.

[37] L. J. Dixon, J. Drummond, T. Harrington, A. J. McLeod, G. Papathanasiou, and M. Spradlin, J. High Energy Phys. 02 (2017) 137.

[38] J. Maldacena, D. Simmons-Duffin, and A. Zhiboedov, J. High Energy Phys. 01 (2017) 013.

[39] J. Golden, A. B. Goncharov, M. Spradlin, C. Vergu, and A. Volovich, J. High Energy Phys. 01 (2014) 091.

[40] J. Drummond, J. Foster, and Ö. Gürdoğan, Phys. Rev. Lett. 120, 161601 (2018).

[41] D. K. Wagner, Discrete Appl. Math. 180, 158 (2015).

[42] I. Gitler and G. Sandoval-Angeles, ENDM 62, 129 (2017).

[43] J. L. Bourjaily, A. J. McLeod, M. Spradlin, M. von Hippel, and M. Wilhelm, Phys. Rev. Lett. 120, 121603 (2018). 
[44] J. L. Bourjaily, Y. H. He, A. J. McLeod, M. von Hippel, and M. Wilhelm, arXiv:1805.09326 [Phys. Rev. Lett. (to be published)].

[45] D. Chicherin, V. Kazakov, F. Loebbert, D. Müller, and D. 1. Zhong, J. High Energy Phys. 05 (2018) 003.

[46] B. Basso and L. J. Dixon, Phys. Rev. Lett. 119, 071601 (2017).

[47] S. Abreu, R. Britto, C. Duhr, and E. Gardi, J. High Energy Phys. 10 (2014) 125.
[48] S. Abreu, R. Britto, C. Duhr, and E. Gardi, J. High Energy Phys. 06 (2017) 114.

[49] S. Abreu, R. Britto, C. Duhr, and E. Gardi, Phys. Rev. Lett. 119, 051601 (2017).

[50] S. Abreu, R. Britto, C. Duhr, and E. Gardi, J. High Energy Phys. 12 (2017) 090.

[51] S. Abreu, R. Britto, C. Duhr, and E. Gardi, Proc. Sci., RADCOR2017 (2018) 002. 